\title{
Artificial Rubber
}

\author{
Another of Nature's Laboratory Secrets Unmasked
}

\section{By Ira Remsen, President of Johns Hopkins University}

$\triangle$ BOU' forty years ago two young chemists an A nounced that they had succeeded in making enough indigo to be pretty sure they' had it. The method used oy them was entirely independent of the indigo plants. The yield was extremely small and there seemed to be no promise in the method of future commercial success. A quarter of a century later or thereabout the manufacturer was supplying the world with artificial indigo and the occupation of the indigo planter, if not gone, was going. And now the chemical world is all agog over rubber. Last year a specimen of artificial rubber was exhibited at Berlin identical in all its properties with the natural substance. At presen there appears, to be sure, to be little prospect of early commercial success along the line suggested, but the results already reached are of such interest as to incite to many efforts to solve the problem.

First a few words concerning rubber. This substance is obtained mainly from South and Central America, though, of late years, owing to the great increase in the demand for it, plantations have been started, esperially in Ceylon and the Nalay Peninsula and Archipelago, and these are now contributing to the world's supply. In 1909 the production of rubber is stated to have been 70,000 tons. It is obtained from various trees as Hevea brasiliensis, Mlanihat Glaziovii, Castilloa elastica, Funtumia elastica, Ficus elastica, Hancornia speciosa, etc. Deep incisions are made near the base of the tree. The liquid flowing from these incisions is hrated to drive off most of the water and the residue is the impure rubber of the market. The valuable con stituent is caoutchouc, a colorless, elastic, amorphous substance. This has long been known but little has been learned in regard to its chemical nature until recently.

In general terms it may be stated that chemists do wo like to work with gummy substances, but prefer things that crystallize, or that boil without decomposition, or that form definite crystallizable products. Now caoutchouc does not crystallize, it does not boil with out decomposition, and until a few years ago it was not known to be capable of yielding definite crystallizable products. Great impetus was given to its study by the discovery of Harries that it forms a well-defined froduct when treated with ozone, and that the ozonide thus obtained decomposes when boiled with water and yields levulinic aldehyde, a substance that can easily be identified.

It has also been shown that caoutchouc forms a so-called nitrosite when treated with nitrous acid, and this nitrosite can also easily be identified. These farts make it possible for the chemist to tel whether he has caoutchouc in band or not and the scientific study of the substance has been materially arientific study of the substance has been materially facts a product had been obtained artificially that was described as "like rubber," but the question whether it was jdentiral with caontrhome or not could not be definitely answered. Now it is possible to determine with certainty whether a substance is or is not caout-

To identify caoutchouc after you get it is one thing to determine what it is is quite another. The first step towards the solution of this problem was taken a number of years ago when Greville Williams showed that when rubber is heated to decomposition a very volatile substance is formed which he called "isoprene." This boils at $36 \mathrm{deg}$. C. and is a hydrocarbon of the formula $\mathrm{C}_{\mathrm{S}} \mathrm{H}_{\mathrm{k}}$. Later Harries found that this same hydrocarbon is formed by the action of moderate heat upon oil of turpentine and other terpenes. This isoprene has come to be the chief factor in the artificial meparation of rubber, for it is this substance which

\section{Arrangement of Laboratory Supplies} By William P. Munge

IN an industrial laboratory, containing some 1,500 bottles of chemicals, it is no small job to keep even the chemicals in order. The helpers upon whom the restoration of the bottles to their places devolves usually have little skill and less interest in things r.hemical. When the rhemicals are arranged by the usual alphabetic method. reasunts are mostly found, and only then after prolonged search in just the place they ought not to be. Owing to the vast difference in size of bottles, to get any efficiency out of shelf space it is necessary to have: at least two or three alphabets. and when the search is completed through one, the others must be searched. when exposed to certain cond
least partly into caoutchouc.

That isoprene readily undergoes change was observed by Tilden who announced in 1892 that one of the prod ucts is caoutchouc. Later he stated that contact wit strong acids as, for example, aqueous hydrochloric acid, causes the change of isoprene into caoutchouc, at least to a small extent. Still later he claims to have discovered that isoprene is changed to caoutchouc spontaneously. He says: "Specimens of isoprene were made from several terpenes in the course of my work, and from several ter'penes in the course of my work, and
some of them I have preserved. I was surprised a few some of them I have preserved. I was surprised a few
weeks ago at finding the contents of the bottle contain ing isoprene from turpentine entirely' changed in appearance. In place of a limpid, colorless liquid, th bottle contained a dense syrup in which were floating several large masses of a solid of a yellowish color Upon examination, this turned out to be India rubber." Harries tried to repeat Tilden's work, but although he kept at it for seven years he was unable to confirm his results. He says: "Tilden must therefore acc dentally have hit upon conditions which caused the polymerization of the isoprene, but, and this is the main point, he did not furnish the proof that he really had produced caoutchouc." Klages had previously publicly stated that it is impossible to produce caoutchouc by the method suggested by Tilden. This was the state of affairs as far as the chemical public was concerned until the summer of 1909. Then Harries received from an English firm a specimen of a substance which it was claimed had been made artificially by a process patented by Dr. Heinemann in England. Harries foun that the substance was in fact caoutchouc, but he did not beliere that it had been made artificially. Heinemann's method consisted in passing acetylene, ethylene, and methyl chloride together through a tube heated to redness. Harries could not make this work and other who tried it also failed.

Lp to this time the experiences of Harries had been anything but encouraging. In November, 1909, he ceived from the Elberfeld Color Factory some spec mens of artificial rubber which had been made from isoprene by a secret process devised by Dr. Fritz Ho mann. This led Harries to take up his own experiments on the subject again, and toward the end of January, 1910, he was in a position to apply for patent. The method devised by him consists in heating isoprene with strong acetic acid in a closed tube. Th isoprene with strong acetic acid in a closed tube. The
product thus obtained is unquestionably caoutchouc. From the scientific point of view the problem of the artificial preparation of caoutchouc is thus solved, but it is a far cry from this to commercial success. Given isoprene in sufficient quantity and at a sufficiently low price and the commercial problem will be solved. At present artificial rubber is almost as precious as th diamond, but, on the whole, the problem is in a mor hopeful state than the indigo problem was for years after the start was made. The manufacturers an those who are carrying on chemical investigations for them are plainly of the opinion that the work is worth while. That great firm, the Baden Aniline and Soda Factory (generally called the "Badische") has taken out a number of patents in this field, and this is most significant. It is this firm that worked out the indig problem and is now supplying the world with artificial indigo. having expended in the preliminary work millions of dollars! It is this firm that is placing the manufacture of artificial nitrates upon a firm scientific basis-probably the most important problem before the world as it involves ultimately the food supply. It may safely be predicted that before many' years have passed rubber will be artificially prepared in quantit sufficient to supply the great and increasing deman

To overcome these obstacles to speedy work, the following system was recently worked out in one in lowing system was recently worked out in one in-
dustrial laboratory: The contents were first rougnly grouped as to size, and then as to nature of contents Beginning on the upper and lower shelves, the containers were placed in rows from front to back. The upper left row was numbered $0-9$, the second row 10-19, the third row $20-29$, etc. The last figure on the fron bottle always being 0 , and if the shelf did not accommodate 10 botrles from front to rear then the numbers corresponding to the missing bottles wer omitted, the next row beginning with the next highe ten. An alphabetical and numerical index was then compiled, giving a little description of the chemica in each bottle (such as C. P. Sticks, commercial powder) and typewritten copies made. for it. Meantime there is no danger of a famine as plantations are springing up to supplement the supply of wild rubber.

As has been pointed out the first thing 10 be done is to get enough cheap isoprene. The only way to do this that appears to be in the least promising is to make it from oil of turpentine. The chief constituent of this oil is a terpene of the composition $\mathrm{C}_{10} \mathrm{H}_{16}$, while isoprene has the composition $\mathrm{C}_{5} \mathrm{H}_{8}$. The immed:ate problem, therefore, is to break down the molecule of the terpene, $\mathrm{C}_{10} \mathrm{H}_{1}$ into simpler molecules of the coinposition, $\mathrm{C}_{;} \mathrm{H}_{8}$. If this could be done easily and cheaply the rubber problem would be solved. In this connection it is of interest to note that, acccrding to an article that has just come to hand, Staudinger and Kleven have succeeded in increasing the yield of isoprene obtained from the terpene, dipentene, by heating to a high temperature the vapor of the terpene under diminished pressure. When the pressure is sufficiently low the yield is about 60 per cent. . This is a very important observation for the purpose in view. Dipentene itself does not, to be sure, occur in nature in very large quantity, but the chief constituent of American oil of turpentine is pinene, a substance of the same composition as dipentene, and pinene is converted into dipentene by heating it to 250 to $270 \mathrm{deg}$. C. The way out may therefore be through turpentine. To recapitulate, turpentine contains pinene. Pinene is converted into dipentene by moderate heat. Dipentene when heated to a high temperature under diminished pressure yields isoprene. Isoprene can be converted into caoutchouc by heating it with concentrated acetic acid and by other methods that need not be described here. There are other hydrocarbons similar to isoprene that undergo changes similar to that by which isoprene is converted into caoutchouc, and there is therefore a prospect of the ultimate preparation of a number of products similar to rubber but differing from it in some respects. The commercial instinct has already led to the patenting of some of the processes involved. This aspect of the subject is, however, or it appears to be, of much less importance than the main problem briefly presented in this article.

In the chemical laboratories connected with the great factories, especially in Germany, where several hundred well trained chemists are frequently engaged in a single factory, no doubt much work is now in progress which has for its object the solution of the artificial rubber problem. This is also true of laboratories not connected with the factories. From time to time an article will appear in a scientific journal or a patent will be announced giving some result of the work. As usual, of the failures and disappointments, which always far outnumber the successes, we shall hear very little. But some day we shall wake up to learn that artificial rubber is on the market and then we shall be told truly that scientific research has scored another brilliant success. In the eyes of the world success will mean commercial success. But it must not be forgotten that this, if and when attained, will be based upon innumerable results that have absolutely no commercial significance. Greville Williams heated rubber and obtained isoprene many years ago. No one dreamed at the time that this simple fact might ultimately become he basis of a great industry. Harries treated caoutchouc with ozone and founded a method for indentifying caoutchouc which has proved of fundamental value in all subsequent work on this subject. Vive want to know what caoutchouc is, whether any one ever devises a method for making it artificially or not, and in the highest sense the knowledge will be just as valuable whether such a method results or not. But that is another story.

When a given chemical is wanted, a reference to the index is essential, but a glance shows what form it is in, and the bottle of that chemical best suited to the work in hand can be at once selected. Further, if a helper, although it may be his first day in the laboratory, is sent after a reagent, and given a number, readily found, instead of an unfamiliar chemical name, the chances are greatly improved of his bringing back what was desired. When the reagents are to be replaced they are much more readily replaced in their correct positions by this method, even y skilled workers.

This method is of wide applicability to medium syed stocks of articles, varying in size and use but wot in name, such as motor and meter parts, construction material, switches, insulators, patterns, etc. 